\title{
Co-culture with lung cancer A549 cells promotes the proliferation and migration of mesenchymal stem cells derived from bone marrow
}

\author{
YUE-MEI ZHANG $^{1}$, ZHI-MING ZHANG ${ }^{2}$, QUAN-LIN GUAN ${ }^{1}$, YONG-QI LIU ${ }^{2,3}$, ZHI-WEI WU ${ }^{2,4}$, JIN-TIAN LI ${ }^{2,3}$, \\ YUN SU ${ }^{1}$, CHUN-LU YAN ${ }^{1}$, YA-LI LUO ${ }^{1}$, JIE QIN $^{1}$, QIAN WANG ${ }^{1}$ and XIAO-DONG XIE \\ ${ }^{1}$ Department of Ophthalmology, First Hospital of Lanzhou University; \\ ${ }^{2}$ Provincial-Level Key Laboratory for Molecular Medicine of Major Diseases and \\ The Prevention and Treatment with Traditional Chinese Medicine Research in Gansu Colleges and Universities; \\ ${ }^{3}$ Key Laboratory of Dunhuang Medical and Transformation, Ministry of Education of The People's Republic of China; \\ ${ }^{4}$ Department of Basic Medical Sciences, Gansu University of Chinese Medicine; ${ }^{5}$ Institute of Genetics, \\ School of Basic Medical Sciences, Lanzhou University, Lanzhou, Gansu 730000, P.R. China
}

Received April 11, 2016; Accepted March 17, 2017

DOI: $10.3892 /$ etm.2017.4909

\begin{abstract}
The initiation and progression of various types of tumors, such as lung neoplasms, are driven by a population of cells with stem cell properties and their microenvironment. Bone marrow mesenchymal stem cells (BM-MSCs) in long-term in vitro culture may exhibit spontaneous changes in stem cell biological properties, including malignant transformations; however, the molecular mechanisms of this have not been fully elucidated. In the present study, a BM-MSC and lung cancer A549 cell co-culture system was utilized to investigate how the tumor microenvironment may spontaneously change the proliferation, migration and differentiation of BM-MSCs. It was demonstrated that the lung cancer A549 microenvironment is able to induce changes in the cell morphology, proliferation, karyotype, cytoskeleton and migration ability of BM-MSCs in vitro. Compared with the control group BM-MSCs, the expression of Ras, phosphorylated-extracellular regulated protein kinases, nuclear factor- $\kappa \mathrm{B}, \mathrm{P} 62$ and $\mathrm{B}$-cell lymphoma 2 (Bcl-2) proteins in groups of co-cultured BM-MSCs increased significantly $(\mathrm{P}<0.05)$ and the expression
\end{abstract}

Correspondence to: Dr Quan-Lin Guan, Department of Ophthalmology, First Hospital of Lanzhou University, 1 Donggang West Road, Lanzhou, Gansu 730000, P.R. China

E-mail: guangquanlin@163.com

Professor Yong-Qi Liu, Provincial-Level Key Laboratory for Molecular Medicine of Major Diseases and The Prevention and Treatment with Traditional Chinese Medicine Research in Gansu Colleges and Universities, 35 Dingxi East Road, Lanzhou, Gansu 730000, P.R. China

E-mail: liuyongqi73@163.com

Key words: bone marrow mesenchymal stem cells, lung cancer A549, proliferation, migration, co-culture of P53, Bcl-2 associated X protein and caspase-3 protein decreased significantly $(\mathrm{P}<0.05)$. The mechanisms responsible for the changes observed in BM-MSCs may be related to abnormal expression of related genes in the ERK signaling pathway.

\section{Introduction}

Malignant tumors are a serious threat to human health and emphasis has been placed on developing novel therapies to treat such malignancies. Tumor biological therapy is the application of modern biological technology and products targeted at tumors to effectively ameliorate the side effects of chemotherapy (1). Bone marrow mesenchymal stem cells (BM-MSCs) cultured in vitro can be induced to proliferate, readily undergo transfection with liposomes carrying exogenous genes, have excellent mobility, are capable of undergoing multilineage differentiation (2) and have low immunogenicity (3). These properties make BM-MSCs effective carrier cells for biological treatments of tumors. Using stem cells as carriers to target drug delivery to malignant tumors alone may reduce the adverse reactions caused by systemic drug distribution (4). Furthermore, using genetically modified BM-MSCs as tumor target gene therapy vectors may enhance anti-tumor effects, providing a novel method for tumor therapy $(5,6)$.

The stem cell niche is the microenvironment in which stem cells exist. The stem cell niche allows interaction between stem cells to regulate their function and fate, and it is a critical factor in stem cell homeostasis. The stem cell niche is able to tightly regulate stem cell self-renewal and proliferation by signal molecules (7). It has been reported that BM-MSCs undergoing long-term in vitro culture may undergo spontaneous changes in terms of their biological characteristics, and may even undergo malignant transformation (8-10). These results suggest that alterations to the cell microenvironment may affect the differentiation and proliferation of stem cells; however, the molecular mechanisms responsible for these 
alterations have not been fully elucidated. It has not yet been reported whether changes to BM-MSC biological characteristics in the lung microenvironment are caused by cytokines, signaling molecules or cellular interactions.

To identify the risk of BM-MSCs undergoing malignant transformation when being used for biological therapies in the tumor microenvironment, the present study utilized a Transwell chamber to co-culture BM-MSCs and lung cancer A549 cells to simulate a tumor microenvironment. From this, it was possible to investigate whether BM-MSCs are able to spontaneously undergo changes in proliferation, migration and differentiation in the tumor microenvironment and whether it was possible to maintain BM-MSC genetic stability in these specific culture conditions. The results of the current study may provide an experimental basis for the clinical application of stem cell therapy.

\section{Materials and methods}

Cells and cell culture. BM-MSCs (Cyagen Biosciences, Inc., Santa Clara, CA, USA) and human lung cancer A549 cells (stored in the Provincial-Level Key Laboratory for Molecular Medicine of Major Diseases and The Prevention and Treatment with Traditional Chinese Medicine Research in Gansu Colleges and Universities, Lanzhou, China) were cultured in complete medium, consisting of Dulbecco's modified Eagle's medium/F12 supplemented with $10 \%$ fetal bovine serum (Hyclone; GE Healthcare Life Sciences, Logan, UT, USA). The culture medium was replenished every 2-3 days. Cell aggregates were typically formed after $24 \mathrm{~h}$ incubation in a humidified chamber at $37^{\circ} \mathrm{C}\left(5 \% \mathrm{CO}_{2}\right)$. Cell aggregates were grown in suspension for 3-5 days before they began to attach to the bottom of the culture bottle. When the cells covered $80-90 \%$ of the bottom of the bottle, they were digested with $0.25 \%$ trypsin to perform a co-culture experiment.

Establishment of co-culture system. A non-contact co-culture system of BM-MSCs and lung cancer A549 cells was established using a Transwell suspension culture chamber with polyethylene terephthalate film combined with a 6-pore plate (Corning 3450; Corning, Inc., Corning, NY, USA). The BM-MSC and A549 groups were groups in which BM-MSC cells and A549 cells were cultured respectively, in independent wells of a 6-well plate. The co-BM-MSC group, including BM-MSCs and A549 cells, co-cultured in the transwell system (BM-MSCs in the upper chamber and A549 cells in the lower chamber). The number of cells seeded per chamber for each group is $5 \times 10^{4}$ cells. Cells were cultured in 6-well plates (Corning 3450) containing the aforementioned complete medium at $37^{\circ} \mathrm{C}(5 \%$ $\mathrm{CO}_{2}$ incubator). Culture medium was replenished every $48 \mathrm{~h}$ and cell growth state was observed under an inverted microscope. On day 7 of culture, cell culture was terminated and single cell suspensions were prepared for detection.

Analysis of cell morphology, cell cycle and cell viability. The aforementioned cells were observed every $24 \mathrm{~h}$ during culture periods to detect changes in cell morphology using an inverted microscope. The partial harvested cell suspensions were fixed at $4^{\circ} \mathrm{C}$ in $70 \%$ ethanol overnight. Propidium iodide (PI) and RNase A were subsequently added (final concentration
$50 \mu \mathrm{g} / \mathrm{ml}$; Beckman Coulter, Inc., Brea, CA, USA) and incubated at $37^{\circ} \mathrm{C}$ for $30 \mathrm{~min}$. Following staining, the cells were washed once or twice with $0.01 \mathrm{~mol} / 1$ phosphate-buffered saline (PBS) and cellular DNA content was measured using a Coulter ${ }^{\circledR}$ Epics ${ }^{\circledR}$ XL ${ }^{\mathrm{TM}}$ Flow Cytometer (Beckman Coulter,Inc.) to analyze the cell cycle, with three replications per group and three repeats per replication. Briefly, cells were washed twice with $0.01 \mathrm{~mol} / 1 \mathrm{PBS}$ and centrifuged for $3 \mathrm{mins}$ at $167.7 \mathrm{x} \mathrm{g}$ at room temperature, then incubated with $70 \%$ precooled ethanol at $4^{\circ} \mathrm{C}$ overnight. The cells were then subjected to $50 \mu \mathrm{g} / \mathrm{ml}$ propidium iodide dye solution (containing $50 \mu \mathrm{g} / \mathrm{ml}$ RNase) and analyzed by $\mathrm{EXPO}^{\mathrm{TM}} 32 \mathrm{ADC}$ version $1.1 \mathrm{C}$ software (Beckman Coulter, Inc.). Cell viability was analyzed using the 3-[4,5-dimethylthiazol-2-yl]-2,5 diphenyltetrazolium bromide (MTT) assay for three wells in each group every day, continuously for 9 days. The BM-MSCs group and A549 group served as the controls. The test was performed with $5 \mathrm{mg} / \mathrm{ml} \mathrm{MTT}$ solution prepared by dissolving MTT powder (Sigma-Aldrich; Merck KGaA, Darmstadt, Germany) in warm PBS $\left(37^{\circ} \mathrm{C}\right)$. Cells $\left(\sim 1 \times 10^{4}\right.$ cells/well) were incubated in 96-microwell plates (Nalge Nunc International, Penfield, NY, USA) at $37^{\circ} \mathrm{C}$ for $24 \mathrm{~h}$. Following incubation, $20 \mu \mathrm{l}$ MTT solution was added to each well and incubated for $4 \mathrm{~h}$ in a $5 \% \mathrm{CO}_{2}$ incubator at $37^{\circ} \mathrm{C}$. Subsequently, the solution was removed and the construct was blotted with filter paper. Finally, $200 \mu 1$ 99.5\% dimethyl sulfoxide (Sigma-Aldrich; Merck KGaA) was added to each well and the plate was shaken for $5 \mathrm{~min}$. For colorimetric readings, $100 \mu \mathrm{l}$ of this solution was transferred to an ELISA plate and the absorbance values of the samples detected were read by an ELISA reader at $570 \mathrm{~nm}$. Using cultivated time as the horizontal axis and the absorbance value as the vertical axis, a growth curve was constructed.

Western blot analysis. Using western blotting, the expression of proteins associated with the extracellular signal-related kinase (ERK) signaling pathway, cell proliferation and apoptosis were analyzed. Total protein was extracted from cells using Radio-Immunoprecipitation Assay cell lysis buffer (Qiagen GmbH, Hilden, Germany). Cells were washed with precooled PBS at $4^{\circ} \mathrm{C}$, then scraped using a disposable cell scraper. RIPA cell lysis buffer (cat. no. R0010; Solarbio Science \& Technology Co., Ltd., Beijing, China) was added to cells and the solution was mixed for $1 \mathrm{~min}$. Cells were collected in $1.5 \mathrm{ml}$ centrifuge tubes. All the aforementioned actions were completed on ice. Cells were stood for $10 \mathrm{~min}$ on ice. Then cells were centrifuged at $12,000 \mathrm{x} \mathrm{g}$ for $10 \mathrm{~min}$ at $4^{\circ} \mathrm{C}$. Supernatant fluid is total protein of cells. The amount of protein was quantified with Bicinchoninic acid Protein quantitative kit (cat. no. P1511; Applygen Technologies Inc., Beijing, China). Briefly, the working reagent and standard solutions containing different concentrations of BSA were prepared according to manufacturer's instructions. The different protein concentrations of the standard solutions were determined using the enzyme marker. Then the standard curve was plotted, enabling the calculation of the protein concentration of the samples. A total of $30 \mu \mathrm{g}$ of protein per lane was separated by $10 \%$ SDS-PAGE and transferred onto polyvinylidene difluoride membranes. The membranes were incubated with blocking buffer containing $5 \%$ skim milk powder and 0.1\% Tween-20 (Bio-Rad Laboratories, Inc., Hercules, CA, 
USA) at $37^{\circ} \mathrm{C}$ for $3 \mathrm{~h}$ and then washed with PBS-Tween-20 three times. Subsequently, the membranes were incubated with primary antibodies P-ERK (cat. no. BS4759; 1:500; Bioworld Technology, Inc., St. Louis Park, MN, USA), nuclear factor (NF)- $\kappa \mathrm{B}$ (cat. no. ab16502; 1:500; Abcam, Cambridge, UK), p53 (cat. no. ZM-0408; 1:500; Santa Cruz Biotechnology, Inc., Dallas, TX, USA), P62 (cat. no. ab91526; 1:500; Abcam), B-cell lymphoma-2 (Bcl-2; cat. no. ab194583; 1:500; Abcam), Bcl-2-associated X protein (Bax; cat. no. ab53154; 1:500; Abcam) and caspase-3 (cat. no. ab59388; 1:500; Abcam) at $4^{\circ} \mathrm{C}$ overnight. After the incubation of primary antibodies, membrane was washed four times with tris-buffered saline with Tween-20 for 10 mins. Following this, the membranes were incubated with horseradish peroxidase-conjugated secondary antibody (goat anti-rabbit immunoglobulin G; cat. no. ab150088; 1:5,000; Abcam). Proteins were quantified using enhanced chemiluminescence advance western blotting detection solution (Abcam) and a gel imaging analysis system (ChemiDoc; Bio-Rad Laboratories, Inc.). $\beta$-actin was used as a reference gene. The grey ratio value between the target protein and reference protein in the same sample was used as the relative expression value of the protein and analyzed using Image $\mathrm{Lab}^{\mathrm{TM}}$ software version 3.0 (Bio-Red Laboratories, Inc.) following three repeats.

Karyotype analysis. Cells at the logarithmic growth phase were coated with $0.2 \mu \mathrm{g} / \mathrm{ml}$ colchicine (cat. no. c8190; Solarbio Science \& Technology Co., Ltd.) and set aside for $3 \mathrm{~h}$. The cells were fixed through low permeability of $0.075 \mathrm{~mol} / \mathrm{l} \mathrm{KCl}$, methanol-acetic acid (3:1), dropped on an ice slide and treated with $0.25 \%$ trypsin for $1 \mathrm{~min}$ at room temperature. Karyotype analysis was performed using an eclipse 80i microscope (Nikon Corporation, Tokyo, Japan) to count 100 mitotic chromosomes using a chromosome image analyzer CytoKtype 6.2.2 (United Biotechnology Corporation, San Jose, CA, USA).

Analysis of cytoskeleton and migration ability of cells. Cell suspensions were placed on a slide, fixed with $4 \%$ paraformaldehyde for $15 \mathrm{~min}$ at room temperature, rinsed with PBS containing 5\% bovine serum albumin (cat. no. A8020; Solarbio Science \& Technology Co., Ltd.) and 0.1\% Triton X-100 and stained with phalloidin-labeled fluorescein isothiocyanate (FITC; cat. no. P5282) and Hoechst (cat. no. H6024; both from Sigma Aldrich, Merck KGaA). The staining procedure was performed following the manufacturer's protocol. Stained slides were placed under a laser scanning confocal microscope to observe the cell cytoskeleton.

Cell migration ability was then investigated using a Millicell $\mu$-Migration Assay kit (EMD Millipore, Billerica, MA, USA), according to the manufacturer's instructions. Briefly, following 7 days of culture, cells from the three groups (A549 group, BM-MSCs group, and co-BM-MSC group) were transplanted into a Millicell $\left(1 \times 10^{5}\right.$ cells/Millicell, with a total volume of $100 \mu \mathrm{l}$ ) of the 24 -well plate. A total of $500 \mu \mathrm{l}$ A549 cell conditioned medium was added into the outer chamber of the Millicell. The aforementioned cells were cultured routinely for $8 \mathrm{~h}$ at $37^{\circ} \mathrm{C}$, and cells that did not migrate were removed using a cotton swab. The 24 -well plate was inverted to air-dry and $500 \mu 10.1 \%$ crystal violet was added at $37^{\circ} \mathrm{C}$ for $30 \mathrm{~min}$. The plate was placed under an inverted microscope to count cells in five fields selected at random. Levels of expression of the cell migration-related gelatinases, matrix metalloproteinase (MMP) were detected by western blot analysis (identical to the aforementioned method, with primary antibodies: MMP-2 (cat. no. BS1236; 1:500; Bioworld Technology, Inc.), MMP-9, (cat. no. P14780; 1:500; Bioworld Technology, Inc.), $\beta$-actin (cat. no. SC-47778; 1:500; Santa Cruz Biotechnology, Inc.) and goat anti-rabbit immunoglobulin $\mathrm{G}$ secondary antibody (cat. no. 150088; 1:5,000; Abcam) to analyze the migration ability of the three groups of cells.

Statistical analysis. Data were expressed as the mean \pm standard deviation. SPSS v. 17.0 software (SPSS, Inc., Chicago, IL, USA) was used to determine statistical significance between groups. For statistical comparisons, parametric analysis of variance with the Turkey's test. $\mathrm{P}<0.05$ was considered to indicate a statistically significant difference.

\section{Results}

Changes in cell morphology. BM-MSCs were uniform in morphology, fibroblast-like, fusiform or spindle-shaped, adherent on the plate, distributed evenly, arranged in an orderly manner with a clear boundary and exhibited swirl-like growth (Fig. 1A). Following co-culture with A549 cells for 7 days, BM-MSCs changed shape, cells became small and short, were arranged in a disorderly manner, exhibited irregular polygon overlapping growth, enlarged nuclei, an uneven nuclear shape and staining, and visible pathological mitotic figures. Similar biological cytomorphological features and growth characteristics were observed between A549 cells and co-BM-MSCs (Fig. 1B-D). These results indicate that co-BM-MSCs have a tendency to undergo malignant transformation (8-10).

Analysis of cell cycle and viability. Flow cytometric analyses demonstrated that, compared with BM-MSCs, the number of cells in the G1 (stationary) phase in the co-BM-MSCs group was lower and the number of cells in the $\mathrm{S}$-(proliferating) phase were significantly higher $(\mathrm{P}<0.05)$. There was a difference in the percentage of cells in the G0/G1 between the BM-MSC group and the co-BM-MSCs group. The percentage of cells in the G0/G1 in BM-MSCs group is $77.8 \pm 5.04 \%$ and in co-BM-MSCs group is $62.57 \pm 6.65 \%$. The percentage of cells in $\mathrm{S}$ in the BM-MSCs group is $9.05 \pm 0.8 \%$ and in co-BM-MSCs group is $23.54 \pm 0.6 \%$ (Fig. 2). Using cultivation time as the horizontal axis and the absorbance value as the vertical axis to draw the growth curve, it was determined that the cell growth curves of the three groups were S-shaped. co-BM-MSCs and BM-MSCs exhibited a slow initial increase in viability from days 1-3 followed by more rapid increases in viability from day 4. The co-BM-MSC group underwent a more rapid increase in viability than the BM-MSC group; however, this increase leveled off after day 8 . The viability of cells in the A549 group was higher than that of cells in the co-BM-MSCs and BM-MSCs groups (Fig. 3). These results demonstrate that the tumor microenvironment created by the A549 cells is able to promote the viability of BM-MSCs.

Changes in the expression of proteins associated with the ERK signaling pathway, proliferation and apoptosis. To investigate 
A

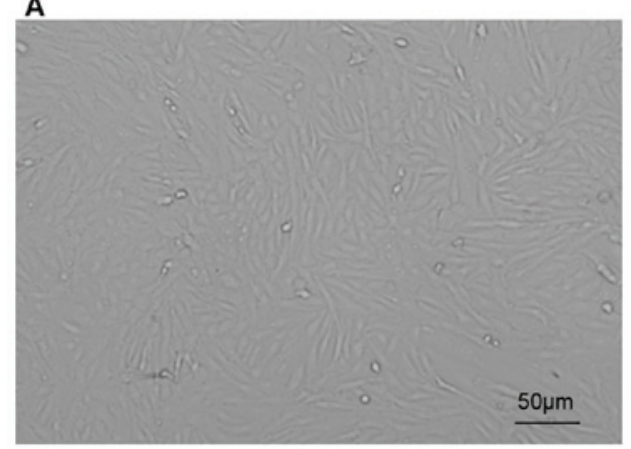

C

$50 \mu \mathrm{m}$

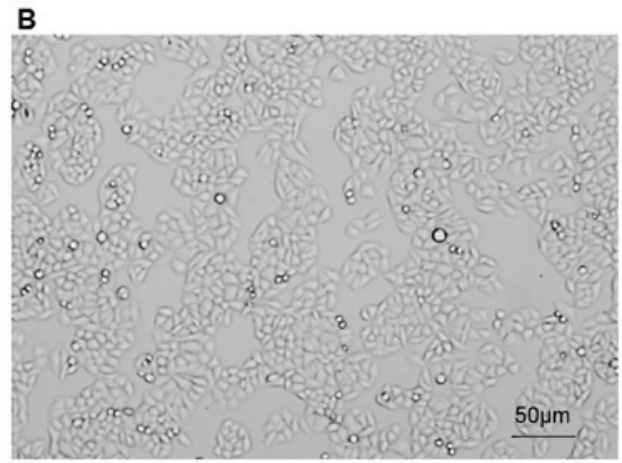

D

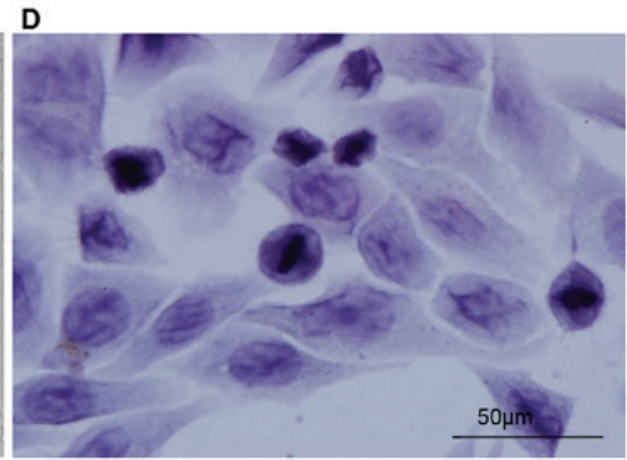

Figure 1. Cell morphology of different groups on day 7 of culture. (A) BM-MSCs were uniform in morphology, fibroblast-like, fusiform or spindle-shaped and adherent on the plate. They were distributed evenly, arranged in an orderly manner with a clear boundary and demonstrated swirl-like growth. Magnification, x100. (B) A549 cells were long fusiform or irregular polygon and arranged in a disorderly manner. Magnification, x100. (C) co-BM-MSCs were short and small, irregular polygon, arranged in a disorderly manner and exhibited overlapping growth. Magnification, x100. (D) co-BM-MSCs stained with hematoxylin staining exhibited enlarged nuclei, an uneven nuclear shape and stain, and exhibited visible pathological mitotic figures. Magnification, x1,000. BM-MSC, bone marrow mesenchymal stem cells; Co-BM-MSCs, BM-MSCs co-cultured with lung cancer A549 cells.
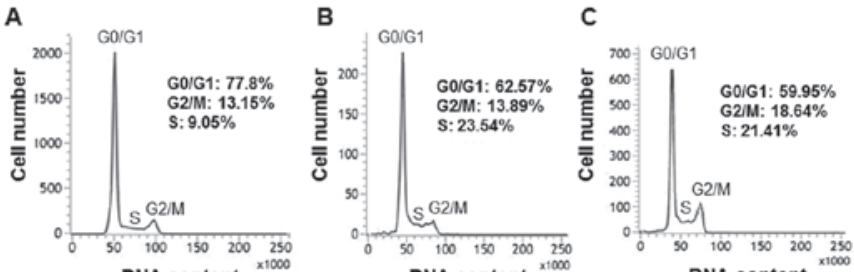

DNA content
Figure 2. Cell cycles of the different groups were measured using a flow cytometer following 7 days culture. (A) BM-MSC cell cycle analysis demonstrated that $77.8 \%$ of cells were in the G0/G1 phase and $9.05 \%$ in the S-phase. (B) Analysis of the co-BM-MSC group demonstrated that $62.57 \%$ of cells were in the G0/G1 and $23.54 \%$ in the S-phase. (C) A549 cell cycle analysis demonstrated that $59.95 \%$ of cells were in the G0/G1 phase and $21.41 \%$ in the S-phase. There was a significant difference between the cell cycle of BM-MSCs and that of co-BM-MSCs $(\mathrm{P}<0.05)$. BM-MSC, bone marrow mesenchymal stem cells; Co-BM-MSCs, BM-MSCs co-cultured with lung cancer A549 cells.

the progression of proliferation and morphological changes of BM-MSCs growing in the tumor microenviroment formed by A549 cells, total proteins were extracted from the three groups of cells following 7 days culture, in order to measure their expression by western blotting. Proteins associated with the ERK signaling pathway were selected to analyze the difference in levels of expression among the three groups of cells. Compared with the BM-MSC group, levels of Ras, P-ERK, $\mathrm{NF}-\kappa \mathrm{B}, \mathrm{P} 62$ and Bcl-2 expression in the co-BM-MSC group were significantly increased $(\mathrm{P}<0.05)$ and levels of $\mathrm{P} 53, \mathrm{Bax}$ and caspase- 3 expression decreased significantly $(\mathrm{P}<0.05$; Fig. 4). Consequently, the changes to the biological properties

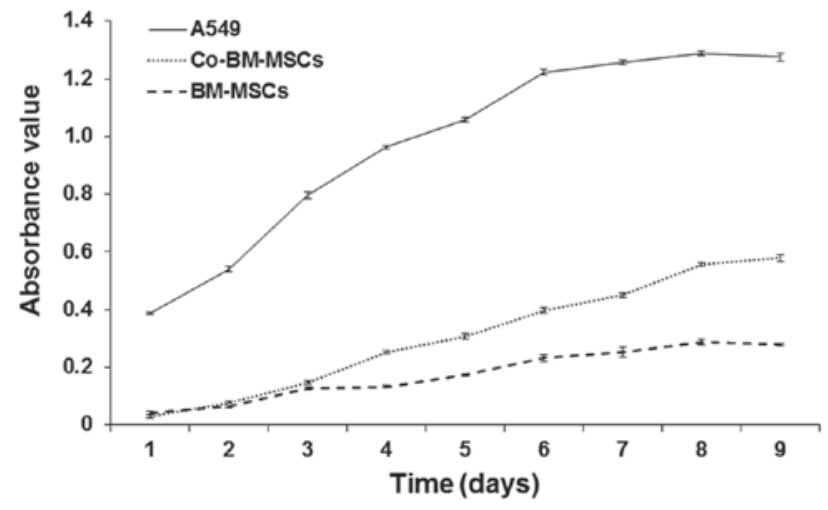

Figure 3. Growth curve profiling of the different cell groups during 9 days culture. MTT analysis demonstrated that the viability of co-BM-MSCs increased more rapidly than that of the BM-MSC group. Data are presented as the mean \pm standard deviation. BM-MSC, bone marrow mesenchymal stem cells; Co-BM-MSCs, BM-MSCs co-cultured with lung cancer A549 cells.

of BM-MSCs following co-culture with A549 cells may be related to abnormal expression of genes associated with the ERK signaling pathway.

Changes in karyotype. Analyzing chromosome karyotypes, which is used to determine cellular genetic structure and type, chromosome number and genetic aberrance, is a basic method of studying cell chromosomes. In the present experiment, the results of the chromosome karyotype analysis determined that BM-MSCs were 46, XX, diploid. By contrast, the co-BM-MSC karyotype was between 46 and 70, hypotriploid, triploid. Part 

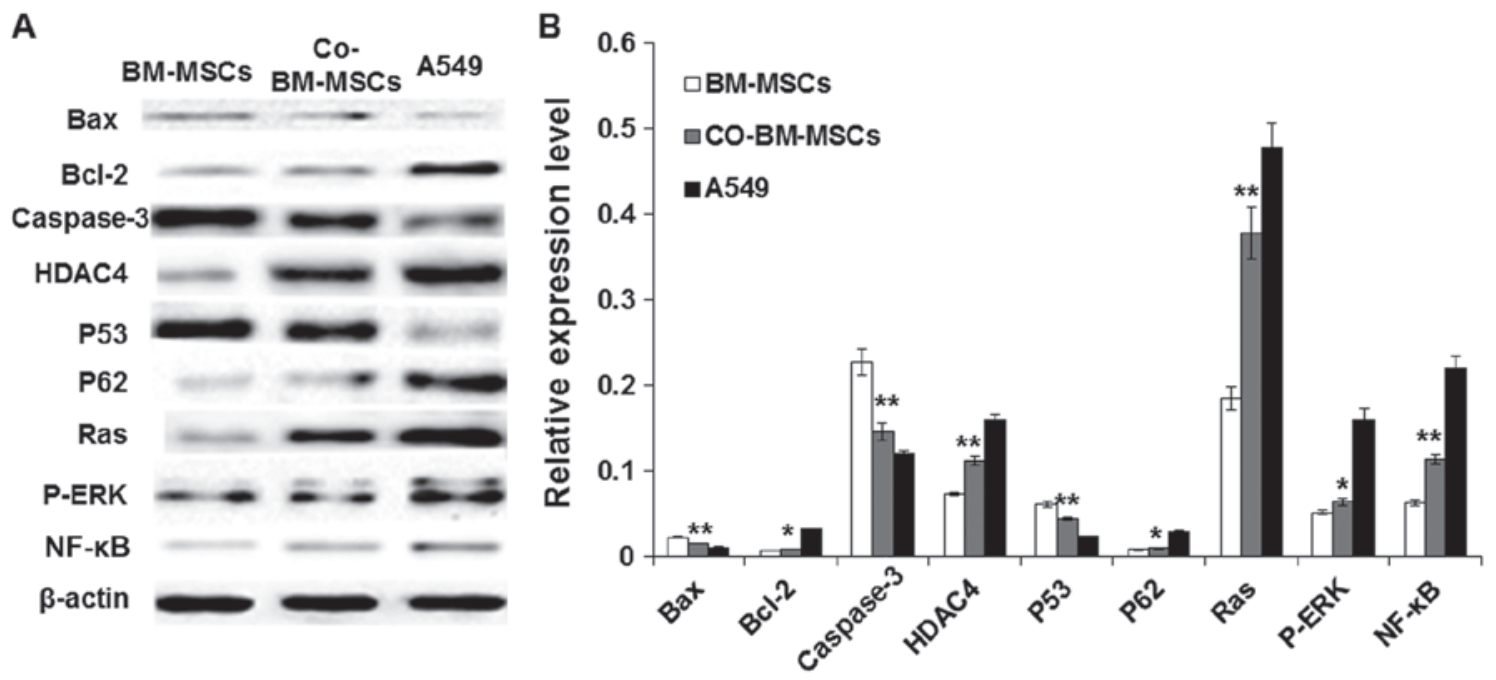

Figure 4. Western blot analysis of the expression of proteins involved in proliferation and differentiation in the different cell groups. (A) Western blotting identified the differential protein expression of Bax, Bcl-2, caspase-3, P53, P62 (c-Myc), Ras, P-ERK and NF-кB in BM-MSCs, co-BM-MSCs and A549 cells. $\beta$-actin was used as a loading control. (B) Relative levels of Bcl-2, Bax, caspase-3, HDAC4, P53, P62 (c-Myc), Ras, P-ERK and NF-kB expression in BM-MSCs, co-BM-MSCs and A549 cells. Compared with the BM-MSC group, levels of Ras, P-ERK, NF- $\mathrm{kB}, \mathrm{P} 62$ and Bcl-2 expression in the co-BM-MSCs group were significantly increased and the levels of P53, Bax and caspase-3 expression decreased significantly. Data are presented as the mean \pm standard deviation of three individual experiments and calculated as relative levels of controls. " $\mathrm{P}<0.01$ and ${ }^{* *} \mathrm{P}<0.05$ vs. the BM-MSC group. BM-MSC, bone marrow mesenchymal stem cells; co-BM-MSCs, BM-MSCs co-cultured with lung cancer A549 cells; Bcl-2, B-cell lymphoma 2; Bax, Bcl-2-associated X protein; P-ERK,

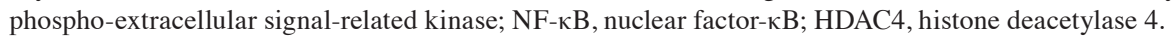

A

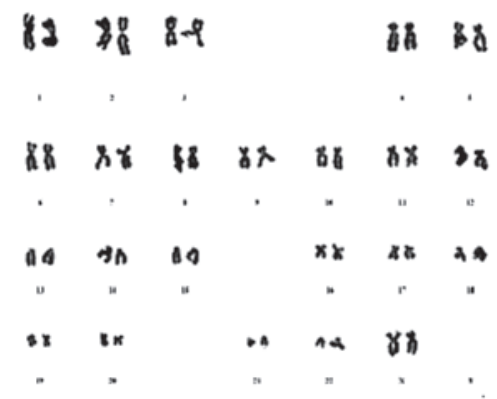

B

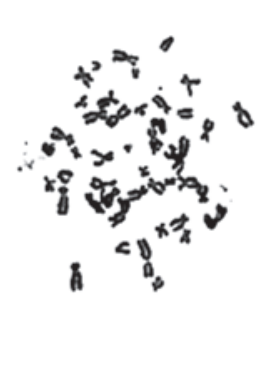

\section{III I8 *\$8 if แI}

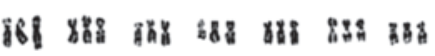
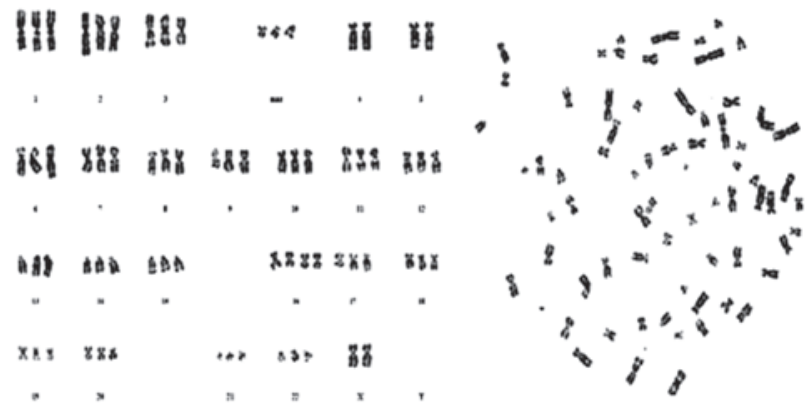

C

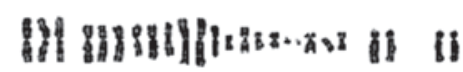

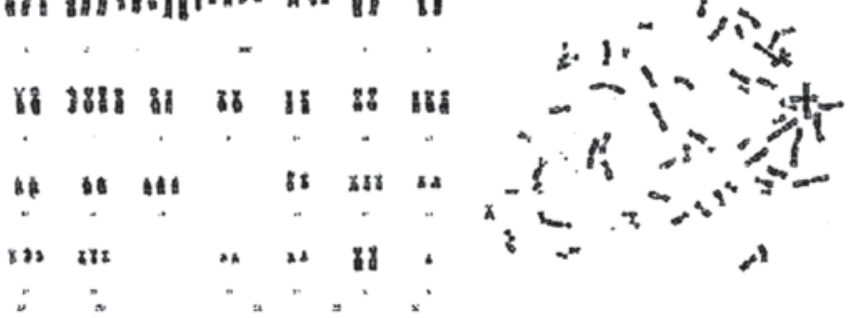

Figure 5. Analysis of cell chromosome karyotypes in the different cell groups. The results of chromosome karyotype analysis demonstrated that the (A) BM-MSC karyotype was 46, XX, diploid, (B) the Co-BM-MSC karyotype was between 46 and 70, hypotriploid, triploid, and (C) the A549 karyotype was between 58 and 83. Part of the Co-BM-MSCs had an aneuploid chromosome number following co-culture, with marked abnormal chromosomes. BM-MSC, bone marrow mesenchymal stem cells; Co-BM-MSCs, BM-MSCs co-cultured with lung cancer A549 cells.

of the co-BM-MSCs cells exhibited an aneuploid chromosome number following co-culture, with obvious abnormal chromosomes (Fig. 5; Table I). These results suggest that, in a tumor microenvironment, BM-MSCs had a malignant tendency.

Study of the cytoskeleton and cell migration. The three groups of cells stained with Hoechst and phalloidin-labeled FITC were placed under a laser scanning confocal microscope to observe the cytoskeleton microfilament. The results demonstrated that the microfilament of BM-MSCs was bundle-like or radial-gathered in the projection and the cytoplasm, whereas that of the co-BM-MSC group was irregular and resembled that of the A549 cells. The microfilament was observed under the microscope (Fig. 6). Compared with the BM-MSCs group, 
A

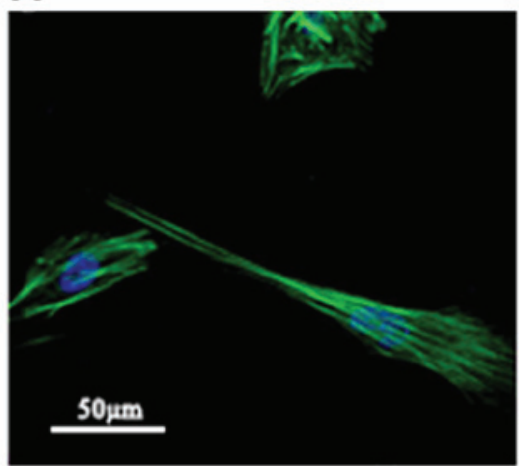

B

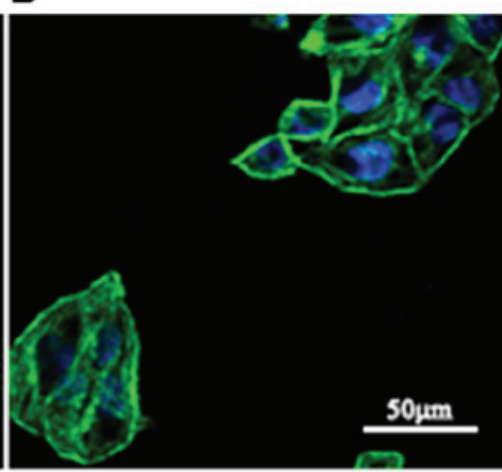

C

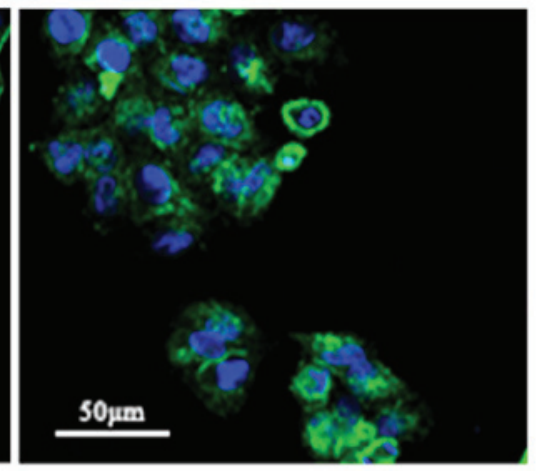

Figure 6. Analysis of cytoskeleton and cell migration. Cells were stained with Hoechst and phalloidin-labeled fluorescein isothiocyanate. The microfilament of (A) BM-MSCs was bundle-like or radial gathered in the projection and the cytoplasm, while that of the (B) Co-BM-MSCs was irregular, similar to that of the (C) A549 cells. Microfilament could be seen under microscope at magnification of x1,000. Scale bar, $50 \mu \mathrm{m}$. BM-MSC, bone marrow mesenchymal stem cells; co-BM-MSCs, BM-MSCs co-cultured with lung cancer A549 cells.
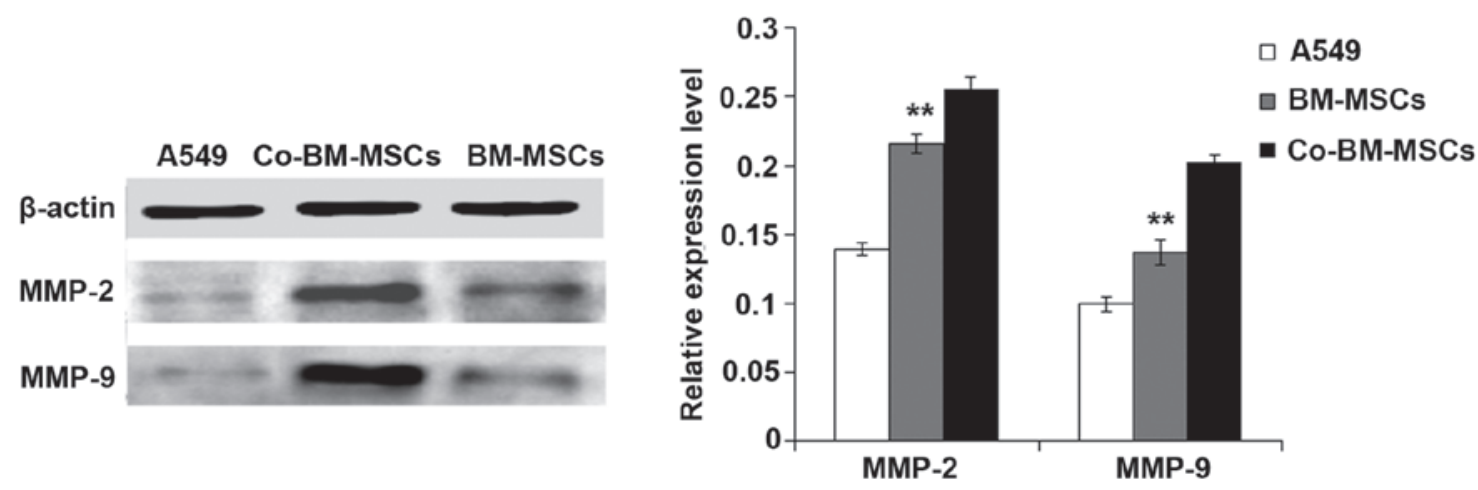

Figure 7. Western blot analysis of the expression of proteins associated with cell migration. Western blotting demonstrated the differential protein expression of MMP-2 and -9 in BM-MSCs, co-BM-MSCs and A549 cells. Compared with the BM-MSC group, the protein expression levels of MMP-2 and -9 were significantly lower compared with the Co-BM-MSC group. $\beta$-actin was used as a loading reference. Data are expressed as the mean \pm standard deviation. ${ }^{* *} \mathrm{P}<0.05$ vs. the BM-MSC group. BM-MSC, bone marrow mesenchymal stem cells; Co-BM-MSCs, BM-MSCs co-cultured with lung cancer A549 cells; MMP, matrix metalloproteinase.

Table I. Chromosome number analysis of the different groups of cells.

\begin{tabular}{|c|c|c|c|}
\hline \multirow[b]{2}{*}{ Variable } & \multicolumn{3}{|c|}{ Group } \\
\hline & BM-MSC & Co-BM-MSC & A549 \\
\hline Chromosome number & 46.0 & $54.4 \pm 8.2^{\mathrm{a}}$ & $62.1 \pm 4.6$ \\
\hline $\begin{array}{l}\text { Rate of chromosome } \\
\text { aberration, \% }\end{array}$ & 0.0 & 66.7 & 100.0 \\
\hline
\end{tabular}

Data are presented as the mean \pm standard deviation where appropriate. ${ }^{a} \mathrm{P}<0.05$ vs. BM-MSC chromosome number. BM-MSC, bone marrow mesenchymal stem cells; Co-BM-MSCs, BM-MSCs co-cultured with lung cancer A549 cells.

the number of cells in the co-BM-MSC group that migrated to the Millicell chamber significantly decreased ( $\mathrm{P}<0.05$; Table II). There was no significant difference in the number of migrated cells in the Millicell chamber between the co-BM-MSC group and A549 group ( $\mathrm{P}>0.05$; Table II). Furthermore, compared with the BM-MSC group, levels of MMP-2 and -9 expression
Table II. Analysis of the number of cells in each group that migrated to the Millicell chamber of the plate.

\begin{tabular}{lc} 
Group & Number of migrating cells \\
\hline BM-MSCs & $89.00 \pm 7.55$ \\
Co-BM-MSCs & $18.67 \pm 3.21^{\mathrm{a}}$ \\
A549 & $12.67 \pm 2.08^{\mathrm{a}}$ \\
\hline
\end{tabular}

Data are presented as the mean \pm standard deviation. ${ }^{\mathrm{a}} \mathrm{P}<0.05$ vs. the BM-MSC group. BM-MSC, bone marrow mesenchymal stem cells; co-BM-MSCs, BM-MSCs co-cultured with lung cancer A549 cells.

were significantly lower compared with the co-BM-MSCs group $(\mathrm{P}<0.05$; Fig. 7$)$.

\section{Discussion}

The cellular microenvironment serves an important role in maintaining normal proliferation, differentiation, metabolism and function. Abnormal changes to microenvironment components may result in the formation of cell lesions (11). The results 
of the present study demonstrate that the viability and number of chromosomes of the BM-MSCs changed abnormally when in the lung cancer A549 cell microenvironment.

Proliferation and differentiation are two important aspects of cellular activities. The proliferation of cells prepares them for differentiation and there is a very close relationship between them. Cell differentiation is inhibited during the proliferative phase, whereas the proliferation ability of differentiated cells is decreased (12). In the present study, the growth curve of the cells of the three groups was identified to be $\mathrm{S}$ type. The co-BM-MSC and BM-MSC groups exhibited a slow increase in viability from days 1-3 and a rapid increase in viability starting from day 4 . The increase in viability was faster in the co-BM-MSC group than the BM-MSC group. After day 8 , increases in cellular viability ceased. The viability of the A549 group was higher than that of the co-BM-MSC and BM-MSC groups.

The cell cycle is the basic process of cell life and refers to the entire life cycle of a cell from the end of one division to the end of the next one (13). Using flow cytometry and PI staining to detect the content of DNA in cells, the cell cycle can be divided into the G1/G0, S- and G2/M phases. G1 phase initiation is the key to cell cycle progression. Whether cells may enter the division cycle and whether the cycle may be completed predominantly depends on its ability to smoothly pass through a series of checkpoints, of which the most important are the G1/S and G2/M transformations (14).

In the present study, analysis of the cell cycle by flow cytometry demonstrated that, compared with the BM-MSC group, the ratio of $\mathrm{G} 1$ phase cells in the co-culture group significantly decreased and those in the S-phase increased markedly. There was a significant difference between the cell cycles of the two groups. The G0 (stationary phase) ratio in the co-culture group was lower than that in the BM-MSC group and the proliferating cell ratio in the S-phase increased markedly compared with that in the BM-MSC group. This demonstrates that the co-culture group had greater proliferation ability than the BM-MSCs group. Compared with the A549 group, there was no significant difference in the G1 and S-phase ratio in the co-culture group, suggesting that the cell cycle of the co-culture group was similar to that of the A549 group. The lung cancer A549 cell microenvironment promoted bone marrow mesenchymal stem cells from the G1 phase to the $\mathrm{S}$-phase and drove the cell cycle transition from $\mathrm{G} 2$ phase to $\mathrm{M}$ phase (15). This change may be associated with the increased number of MSCs, which were no longer able to undergo multi-directional differentiation (16).

Chromosomal changes include alterations to chromosome number and structure. Compared with gene mutations, aneuploidy is more relative to cancer. Cytogenetic data have demonstrated that almost all tumor cells are represented as aneuploid karyotypes $(17,18)$. Li et al (19) proposed the aneuploid hypothesis of cancer, where the normal gene expression imbalance caused by aneuploidy is the root of tumor formation, and aneuploidy rather than other mutations is able to provide a more reasonable explanation of tumor cells in a variety of phenotypes $(19,20)$. In the experiment, regular karyotype analysis demonstrated that total cell chromosome number in the control group was 46, while in the experimental group, cell chromosome numbers ranged from 46-70 $(19,20)$. The results illustrate that the tumor microenvironment may stimulate aneuploidy in BM-MSC chromosomes.

A key problem with stem cell transplantation is how to regulate stem cell migration, proliferation and differentiation following transplantation. Previous studies have demonstrated that signals for the survival, proliferation and apoptosis of various cells are transduced through the activation of mitogen-activated protein kinase (MAPK) and the phosphoinositide 3-kinase/protein kinase B signaling pathways (21-23). MAPK is a widely distributed intracellular protein kinase that contains serine/threonine residues (21-23). MAPKs are involved in various physiological activities, including cell growth, division, differentiation and death, which regulate a series of complex biological functions. The activation of the ERK1/2 pathway is triggered by cell surface receptors. In cultured cells, the ERK pathway is associated with cell proliferation, and whether it promotes or inhibits proliferation depends on the cells, as the ERK pathway activates different genes in different cellular environments. The ERK pathway has different effects due to different activation times and strengths (22). In certain cells, the ERK pathway may also mediate differentiation signals. The ERK pathway is involved in the regulation of the cell cycle and its activation may be anti-apoptotic (24), leading to unlimited cell proliferation. The P38 MAPK pathway is associated with the proliferative response induced by cytokines and inhibiting the P38 MAPK pathway may inhibit the induction of cell proliferation by interleukin (IL)-2 and IL-7 (25). Overall, pathways related to apoptosis, such as the P38 MAPK pathway, and catabolic signals together determine whether caspase activation leads to irreversible apoptotic processes (26). Research has demonstrated that the P38 MAPK pathway is related to BM-MSC proliferation (27). In the present study, P-ERK in the P38 MAPK pathway was analyzed by western blot analysis. These results indicate that BM-MSCs co-cultured with lung cancer A549 cells may spontaneously change their cell proliferation ability, possibly via the P38 MAPK pathway.

$\mathrm{NF}-\kappa \mathrm{B}$ is a fast response transcription factor readily detected in eukaryotic cells and also serves an important role in proliferation, differentiation, apoptosis and autophagic cells. Research suggests that the damage factor of NF- $\mathrm{NB}$ activation may produce pro-apoptotic factors, including p53, c-Myc and cyclin D1 in neural cells, leading to the induction of apoptosis $(4,28,29)$. The tumor suppressor p53 is a key mediator of apoptosis. P53 is the target gene of NF- $\kappa \mathrm{B}$. Nuclear translocation of $N F-\kappa B$ may upregulate p53 expression and inhibition of NF- $\kappa \mathrm{B}$ nuclear translocation may downregulate p53 expression; thus NF- $\kappa \mathrm{B}$ may serve an anti-toxicity role (30-32). P53 regulates a variety of genes and some of them may affect mitochondrial genes, thereby affecting cellular apoptosis. P53 may also be transferred to the mitochondria, affecting depolarization of the mitochondrial membrane, releasing pro-apoptotic factors and inducing apoptosis. Furthermore, p53 is an important transcription regulator of cell proliferation, not only in tumor cells, but also in stem cells (33). Research has demonstrated that there are regulatory pathways in which p53 is not only the cell genome supervisor but also the protector for the proliferation and differentiation of cell precursors (32).

Apoptosis, as in cell proliferation and differentiation, serves an important role in maintaining cellular homeostasis as it is 
the process of programmed cell death induced by physical and chemical factors (34). Apoptosis is regulated by a series of genes, such as those from the Bcl-2 gene family. High expression of $\mathrm{Bcl}-2$ protein may protect cells from apoptosis (35), whereas Bax induces cellular apoptosis. Bax and Bcl-2 therefore regulate cell apoptosis positively and negatively, respectively, and their ratio determines whether cells undergo apoptosis or not.

In the present study, the viability of BM-MSCs co-cultured with human lung adenocarcinoma A549 cells was greater than that of the control group BM-MSCs. Western blotting demonstrated that levels of NF- $\mathrm{BB}, \mathrm{Bcl}-2$, Bax, p53, caspase-3 and Ras expression in co-cultured BM-MSCs were between the levels of those in the A549 and BM-MSCs groups. These results indicate that the co-culture tumor microenvironment may alter gene expression via molecular pathways, resulting in the cell evading physiological apoptosis and leading to abnormal cellular proliferation. The tumor suppressor gene p53 and the oncogene Ras are key genes involved in cell signal transduction pathways, which determine cellular fates (36). P53 and Ras regulate key cellular functions, including cell proliferation, programmed cell death, cell movement, inflammation and angiogenesis (37). At present, it has been demonstrated that hundreds of proteins interact with p53. Similarly, Ras has a complex regulatory network. Although p53 and Ras have different molecular pathways, together they control key cellular procedures (38). A study by Buganim et al (39) demonstrated that activation of Ras and inactivation of $\mathrm{p} 53$ were able to induce expression of the NF- $\kappa \mathrm{B}$ gene and a large number of studies have demonstrated that the regulation of NF- $\mathrm{kB}$ by p53 and Ras is important in carcinogenesis $(40,41)$. Such findings suggest that there are synergistic effects between p53 and Ras, and both direct and indirect regulatory interactions and networks may exist between them. Judging from the results of the present experiment, the tumor microenvironment may result in the abnormal proliferation of MSCs due to the synergism between $\mathrm{p} 53$ and Ras.

The key to successful stem cell transplantation is the successful migration, proliferation and the genetic stability of stem cells following transplantation (42). The response of cells to outside stimulation is through interactions between different signaling pathways. No path exists independently in cell reactions and each pathway may integrate with many other pathways. The intensity of the regulation and the interplay between signaling pathways decides the fate of the associated cells (1). The results of the present experiment indicate that the tumor microenvironment is able to induce spontaneous changes in the proliferation and migration ability of BM-MSCs. However, the exact molecular mechanism has not been fully elucidated and the application of BM-MSCs in biological treatment requires further investigation.

The tumor microenvironment is a complex system that consists of numerous stromal cells. Tumor cells, through the production of growth factors and proteases, regulate the tumor stromal environment and may also activate various types of cells in tumor stroma at the same time (43). Following activation, stromal cells secrete insulin-like growth factor-1, hepatocyte growth factor and other cytokines, which promote the malignant transformation of cells (8).

In conclusion, the in vitro lung cancer A549 microenvironment may induce BM-MSCs to undergo changes in cell morphology, proliferation, karyotype, cytoskeleton and migration ability. The underlying mechanisms for these changes may be related to abnormal expression of related genes in the ERK signaling pathway and apoptosis.

\section{Acknowledgements}

The present study was supported by the Outstanding Youth Fund Projects of Gansu Province (grant no. 1308RJDA008) and the National Natural Science Foundation of China (grant nos. 81360588 and 81473457).

\section{References}

1. Attar R, Sajjad F, Qureshi MZ, Tahir F, Hussain E, Fayyaz S and Farooqi AA: TRAIL based therapy: Overview of mesenchymal stem cell based delivery and miRNA controlled expression of TRAIL. Asian Pac J Cancer Prev 15: 6495-6497, 2014.

2. Nakamizo A, Marini F, Amano T, Khan A, Studeny M, Gumin J, Chen J, Hentschel S, Vecil G, Dembinski J, et al: Human bone marrow-derived mesenchymal stem cells in the treatment of gliomas. Cancer Res 65: 3307-3318, 2005.

3. Maitra B, Szekely E, Gjini K, Laughlin MJ, Dennis J, Haynesworth SE and Koç ON: Human mesenchymal stem cells support unrelated donor hematopoietic stem cells and suppress T-cell activation. Bone Marrow Transplant 33: 597-604, 2004.

4. Gutova M, Shackleford GM, Khankaldyyan V, Herrmann KA, Shi XH, Mittelholtz K, Abramyants Y, Blanchard MS, Kim SU, Annala AJ, et al: Neural stem cell-mediated CE/CPT-11 enzyme/prodrug therapy in transgenic mouse model of intracerebellar medulloblastoma. Gene Ther 20: 143-150, 2013.

5. Nakamura K, Ito Y, Kawano Y, Kurozumi K, Kobune M, Tsuda H, Bizen A, Honmou O, Niitsu Y and Hamada H: Antitumor effect of genetically engineered mesenchymal stem cells in a rat glioma model. Gene Ther 11: 1155-1164, 2004.

6. Studeny M, Marini FC, Dembinski JL, Zompetta C, Cabreira-Hansen M, Bekele BN, Champlin RE and Andreeff M: Mesenchymal stem cells: Potential precursors for tumor stroma and targeted-delivery vehicles for anticancer agents. J Natl Cancer Inst 96: 1593-1603, 2004

7. Jones DL and Wagers AJ: No place like home: Anatomy and function of the stem cell niche. Nat Rev Mol Cell Biol 9: 11-21, 2008.

8. Kalluri R and Zeisberg M: Fibroblasts in cancer. Nat Rev Cancer 6: 392-401, 2006.

9. Miura M, Miura Y, Padilla-Nash HM, Molinolo AA, Fu B, Patel V, Seo BM, Sonoyama W, Zheng JJ, Baker CC, et al: Accumulated chromosomal instability in murine bone marrow mesenchymal stem cells leads to malignant transformation. Stem Cells 24: 1095-1103, 2006.

10. Rosland GV, Svendsen A, Torsvik A, Sobala E, McCormack E, Immervoll H, Mysliwietz J, Tonn JC, Goldbrunner R, Lønning PE, et al: Long-term cultures of bone marrow-derived human mesenchymal stem cells frequently undergo spontaneous malignant transformation. Cancer Res 69: 5331-5339, 2009.

11. Scadden DT: The stem-cell niche as an entity of action. Nature 441: 1075-1079, 2006.

12. Peister A, Mellad JA, Larson BL, Hall BM, Gibson LF and Prockop DJ: Adult stem cells from bone marrow (MSCs) isolated from different strains of inbred mice vary in surface epitopes, rates of proliferation, and differentiation potential. Blood 103: 1662-1668, 2004.

13. Nurse P: A long twentieth century of the cell cycle and beyond. Cell 100: 71-78, 2000 .

14. King RW, Deshaies RJ, Peters JM and Kirschner MW: How proteolysis drives the cell cycle. Science 274: 1652-1659, 1996.

15. Bergfeld SA, Blavier L and Declerck YA: Bone marrow-derived mesenchymal stromal cells promote survival and drug resistance in tumor cells. Mol Cancer Ther 13: 962-975, 2014.

16. Hartwell LH and Weinert TA: Checkpoints: Controls that ensure the order of cell cycle events. Science 246: 629-634, 1989.

17. Mitelman F, Mertens F and Johansson B: A breakpoint map of recurrent chromosomal rearrangements in human neoplasia. Nat Genet 15 Spec No: 417-474, 1997.

18. Nowell PC: Foundations in cancer research. Chromosomes and cancer: The evolution of an idea. Adv Cancer Res 62: 1-17, 1993. 
19. Li R, Sonik A, Stindl R, Rasnick D and Duesberg P: Aneuploidy vs. gene mutation hypothesis of cancer: Recent study claims mutation but is found to support aneuploidy. Proc Natl Acad Sci USA 97: 3236-3241, 2000.

20. Valind A, Jin Y, Baldetorp B and Gisselsson D: Whole chromosome gain does not in itself confer cancer-like chromosomal instability. Proc Natl Acad Sci USA 110: 21119-21123, 2013.

21. Osaki M, Oshimura M and Ito H: PI3K-Akt pathway: Its functions and alterations in human cancer. Apoptosis 9: 667-676, 2004.

22. Rubinfeld $H$ and Seger R: The ERK cascade: A prototype of MAPK signaling. Mol Biotechnol 31: 151-174, 2005.

23. Squires MS, Hudson EA, Howells L, Sale S, Houghton CE, Jones JL, Fox LH, Dickens M, Prigent SA and Manson MM: Relevance of mitogen activated protein kinase (MAPK) and phosphotidylinositol-3-kinase/protein kinase B (PI3K/PKB) pathways to induction of apoptosis by curcumin in breast cells. Biochem Pharmacol 65: 361-376, 2003.

24. Widmann C, Gerwins P, Johnson NL, Jarpe MB and Johnson GL: MEK kinase 1, a substrate for DEVD-directed caspases, is involved in genotoxin-induced apoptosis. Mol Cell Biol 18 2416-2429, 1998

25. Wang Y, Chen AD, Lei YM, Shan GQ, Zhang LY, Lu X and Chen ZL: Mannose-binding lectin inhibits monocyte proliferation through transforming growth factor- $\beta 1$ and $\mathrm{p} 38$ signaling pathways. PLoS One 8: e72505, 2013

26. Wagner EF and Nebreda AR: Signal integration by JNK and p38 MAPK pathways in cancer development. Nat Rev Cancer 9 : 537-549, 2009.

27. Forte G, Minieri M, Cossa P, Antenucci D, Sala M, Gnocchi V, Fiaccavento R, Carotenuto F, De Vito P, Baldini PM, et al: Hepatocyte growth factor effects on mesenchymal stem cells: Proliferation, migration, and differentiation. Stem Cells 24 23-33, 2006

28. Bui NT, Livolsi A, Peyron JF and Prehn JH: Activation of nuclear factor kappaB and Bcl-x survival gene expression by nerve growth factor requires tyrosine phosphorylation of IkappaBalpha. J Cell Biol 152: 753-764, 2001.

29. Herrmann O, Baumann B, de Lorenzi R, Muhammad S Zhang W, Kleesiek J, Malfertheiner M, Köhrmann M, Potrovita I, Maegele I, et al: IKK mediates ischemia-induced neuronal death. Nat Med 11: 1322-1329, 2005

30. Liang ZQ, Wang X, Li LY, Wang Y, Chen RW, Chuang DM, Chase TN and Qin ZH: Nuclear factor-kappaB-dependent cyclin D1 induction and DNA replication associated with $\mathrm{N}$-methyl-D-aspartate receptor-mediated apoptosis in rat striatum. J Neurosci Res 85: 1295-1309, 2007.

31. Zhang XD, Wang Y, Wang Y, Zhang X, Han R, Wu JC, Liang ZQ, Gu ZL, Han F, Fukunaga K and Qin ZH: p53 mediates mitochondria dysfunction-triggered autophagy activation and cell death in rat striatum. Autophagy 5: 339-350, 2009
32. Zhao $\mathrm{T}$ and $\mathrm{Xu} \mathrm{Y}$ : $\mathrm{p} 53$ and stem cells: New developments and new concerns. Trends Cell Biol 20: 170-175, 2010.

33. Das S, Raj L, Zhao B, Bernstein A, Aaronson SA and Lee SW: Hzf, a key modulator of p53 mediated transcription, functions as a critical determinant of cell survival and death upon genotoxic stress. Cell 130: 624-637, 2007.

34. Haanen C and Vermes I: Apoptosis: Programmed cell death in fetal development. Eur J Obstet Gynecol Reprod Biol 64: 129-133, 1996

35. Chao DT and Korsmeyer SJ: BCL-2 family: Regulators of cel death. Annu Rev Immunol 16: 395-419, 1998.

36. Sakai E, Rikimaru K, Ueda M, Matsumoto Y, Ishii N, Enomoto S, Yamamoto $\mathrm{H}$ and Tsuchida N: The p53 tumor-suppressor gene and ras oncogene mutations in oral squamous-cell carcinoma. Int J Cancer 52: 867-872, 1992.

37. Hanahan D and Weinberg RA: Hallmarks of cancer: The next generation. Cell 144: 646-674, 2011

38. El-Serafi MM, Bahnassy AA, Ali NM, Eid SM, Kamel MM, Abdel-Hamid NA and Zekri AR: The prognostic value of c-Kit, K-ras codon 12, and p53 codon 72 mutations in Egyptian patients with stage II colorectal cancer. Cancer 116: 4954-4964, 2010

39. Buganim Y, Solomon H, Rais Y, Kistner D, Nachmany I, Brait M, Madar S, Goldstein I, Kalo E, Adam N, et al: p53 Regulates the Ras circuit to inhibit the expression of a cancer-related gene signature by various molecular pathways. Cancer Res 70: 2274-2284, 2010

40. Enomoto T, Fujita M, Inoue M, Ric JM, Nakajima R, Tanizawa O and Nomura T: Alterations of the p53 tumor suppressor gene and its association with activation of the c-K-ras-2 protooncogene in premalignant and malignant lesions of the human uterine endometrium. Cancer Res 53: 1883-1888, 1993.

41. Bardeesy N, Bastian BC, Hezel A, Pinkel D, DePinho RA and Chin L: Dual inactivation of RB and p53 pathways in RAS-induced melanomas. Mol Cell Biol 21: 2144-2153, 2001.

42. Park KI, Hack MA, Ourednik J, Yandava B, Flax JD, Stieg PE, Gullans S, Jensen FE, Sidman RL, Ourednik V and Snyder EY: Acute injury directs the migration, proliferation, and differentiation of solid organ stem cells: Evidence from the effect of hypoxia-ischemia in the CNS on clonal 'reporter' neural stem cells. Exp Neurol 199: 156-178, 2006.

43. Madjd Z, Gheytanchi E, Erfani E and Asadi-Lari M: Application of stem cells in targeted therapy of breast cancer: A systematic review. Asian Pac J Cancer Prev 14: 2789-2800, 2013. 